$>$ Les jonctions communicantes (gap junctions) sont des structures membranaires permettant la diffusion intercellulaire de petites molécules (ions, sucres, acides aminés, nucléotides...). La perte de leur fonction, fréquemment induite par des promoteurs de tumeur et associée au phénotype tumorigénique, a fait supposer que les jonctions communicantes étaient impliquées dans le processus de cancérogenèse. Plus récemment, cette hypothèse a été confortée par le fait que le rétablissement de la communication jonctionnelle intercellulaire s'accompagne d'un effet suppresseur de tumeur spécifique. Malgré ces données, plusieurs zones d'ombre subsistent, parmi lesquelles le mode de régulation de l'effet suppresseur et la véritable implication des jonctions communicantes dans la cancérogenèse humaine. Répondre à ces interrogations est d'importance, puisque les jonctions communicantes pourraient être un paramètre à considérer en terme d'efficacité pour certaines thérapies géniques anticancéreuses, voire même pour certaines chimiothérapies. <

\section{Jonctions communicantes et cancer : implications et perspectives}

Marc Mesnil

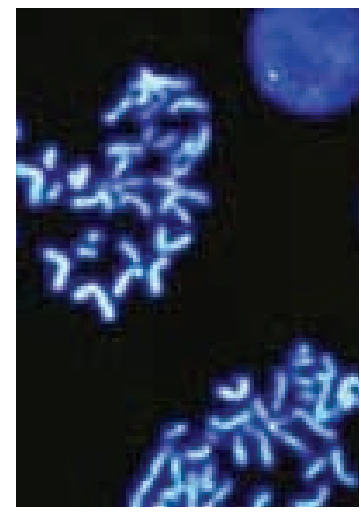

Équipe Communication jonctionnelle, CNRS UMR 6558, Laboratoire des Biomembranes et Signalisation cellulaire, Université de Poitiers, 40, avenue du Recteur Pineau, 86022 Poitiers Cedex, France.

sés de petite taille, marc.mesnil@univ-poitiers.fr dont certains pourraient agir sur la réplication cellulaire et rendre compte de l'inhibition de contact [3]. Par ailleurs, la nature des molécules capables de diffuser par les jonctions communicantes suggère que ces dernières possèdent un rôle fondamental, souligné par leur présence dans la quasitotalité des cellules, à quelques exceptions près : cellules sanguines circulantes, certains neurones, spermatozoïdes et cellules musculaires squelettiques matures.

L'inhibition de contact, qui désigne l'arrêt de prolifération de cellules à confluence, décrite dès les premières cultures cellulaires [1], laissait présager que le contact intercellulaire favorisait la transmission de signaux antimitotiques de cellule à cellule. À l'opposé, les cellules cancéreuses, ayant souvent perdu cette propriété d'inhibition de contact, faisaient figures d'exception, et leur étude pouvait alors procurer des indices susceptibles d'élucider ce phénomène.

Parmi les différents moyens de communiquer dont disposent les cellules, les jonctions communicantes (gap junctions) ont vite été suspectées d'être les responsables de la transmission des signaux antimitotiques. Par l'intermédiaire des canaux intercellulaires constitués de connexines qu'elles contiennent (Figure 1), les jonctions communicantes assurent l'échange de compoLes connexines $(C x)$ constituent une famille multigénique dont les membres se différencient surtout par la longueur et la séquence de leur terminaison carboxyle. Ces variations permettent de les classer selon une nomenclature dérivée de leur poids moléculaire (de la plus petite des connexines de $23 \mathrm{kDa}$ ou $\mathrm{C} \times 23$, à la plus grande de $62 \mathrm{kDa}$ ou Cx62) (Figure 1B) [4]. On ignore actuellement le rôle physiologique exact joué par ces différentes connexines. Leur spécificité d'expression et de perméabilité est néanmoins en faveur d'une implication dans des processus d'organogenèse et de maintien de fonctions tissulaires. Ces implications semblent confirmées par l'existence de maladies associées à des altérations de certaines connexines ( $C \times 32$ et neuropathie de Charcot-Marie-Tooth liée au chromosome X, Cx26 et surdité...) [4], et par le phénotype de souris transgé- 
niques dont le gène d'un type particulier de connexines a été invalidé (Tableau I).

\section{Prémices d'une implication}

C'est en étudiant des cellules cancéreuses que W.R. Loewenstein a suggéré, dès les années 1960, que la communication jonctionnelle participait à la régulation de la prolifération cellulaire (Figure 2). Il avait observé que des cellules de tumeurs du foie chez le rat sont, à l'inverse des hépatocytes normaux, incapables d'échanges ioniques. Or des observations similaires avaient été effectuées lors de l'étude de tumeurs thyroïdiennes chez les rongeurs, et de tumeurs de l'estomac chez l'homme [5]. W.R. Loewenstein stipula donc que l'absence de communication jonctionnelle était une caractéristique des cellules cancéreuses participant au dérèglement de la multiplication cellulaire [6]. Cette caractéristique avait à l'époque plus valeur de postulat que de vérité scientifiquement démontrée car la bibliographie n'était pas riche de travaux dans ce domaine, elle était même émaillée d'exceptions [5]. Pourtant, I'hypothèse fut acceptée, probablement du fait qu'il était facile d'établir une relation de cause à effet entre une «absence de communication directe» et un «comportement asocial» des cellules tumorales au sein d'un tissu strictement organisé.

En 1979, au moment où W.R. Loewenstein publiait une revue sur le sujet [5], deux équipes confortaient son hypothèse en montrant qu'un promoteur tumoral, le

Figure 1. Structure moléculaire des jonctions communicantes.

A. Représentation schématique d'une jonction communicante, enchâssée dans les bicouches lipidiques des membranes plasmiques de deux cellules en contact (d'après [2]). Les canaux intercellulaires de la jonction communicante sont constitués par la juxtaposition de deux demi-canaux (ou connexons) provenant chacun d'une des deux cellules adjacentes. Chaque connexon est lui-même formé de six connexines arrangées de telle façon qu'elles ménagent un pore central (certains sont visibles ici en coupe) permettant le passage, de cytoplasme à cytoplasme, de molécules d'un poids moléculaire inférieur à $1200 \mathrm{Da}$ (seconds messagers : calcium, inositol triphosphate, adénosine monophosphate cyclique ; nucléotides ; oses ; ions ; acides aminés) [3]. B. Structure moléculaire des connexines. Les connexines appartiennent à une famille multigénique d'une vingtaine de membres. Toutes semblent posséder la même topologie, particulière, qui se distingue par trois parties bien définies. La partie cytoplasmique est constituée des deux extrémités amino- et carboxy-terminales et d'une boucle intracellulaire (I). Cette partie est la plus variable en séquence d'acides aminés et en longueur (surtout au niveau des zones bleues, par opposition aux zones vertes relativement conservées d'un type de connexine à l'autre), et facilite la caractérisation des connexines selon leur poids moléculaire. Des sites de phosphorylation différents selon les connexines sont également localisés le long de la terminaison carboxyle et permettent des régulations fonctionnelles variées et rapides par des kinases cytoplasmiques. Par ailleurs, des sites d'interaction potentielle avec d'autres protéines cytoplasmiques sont également présents sur certaines connexines. La partie membranaire est

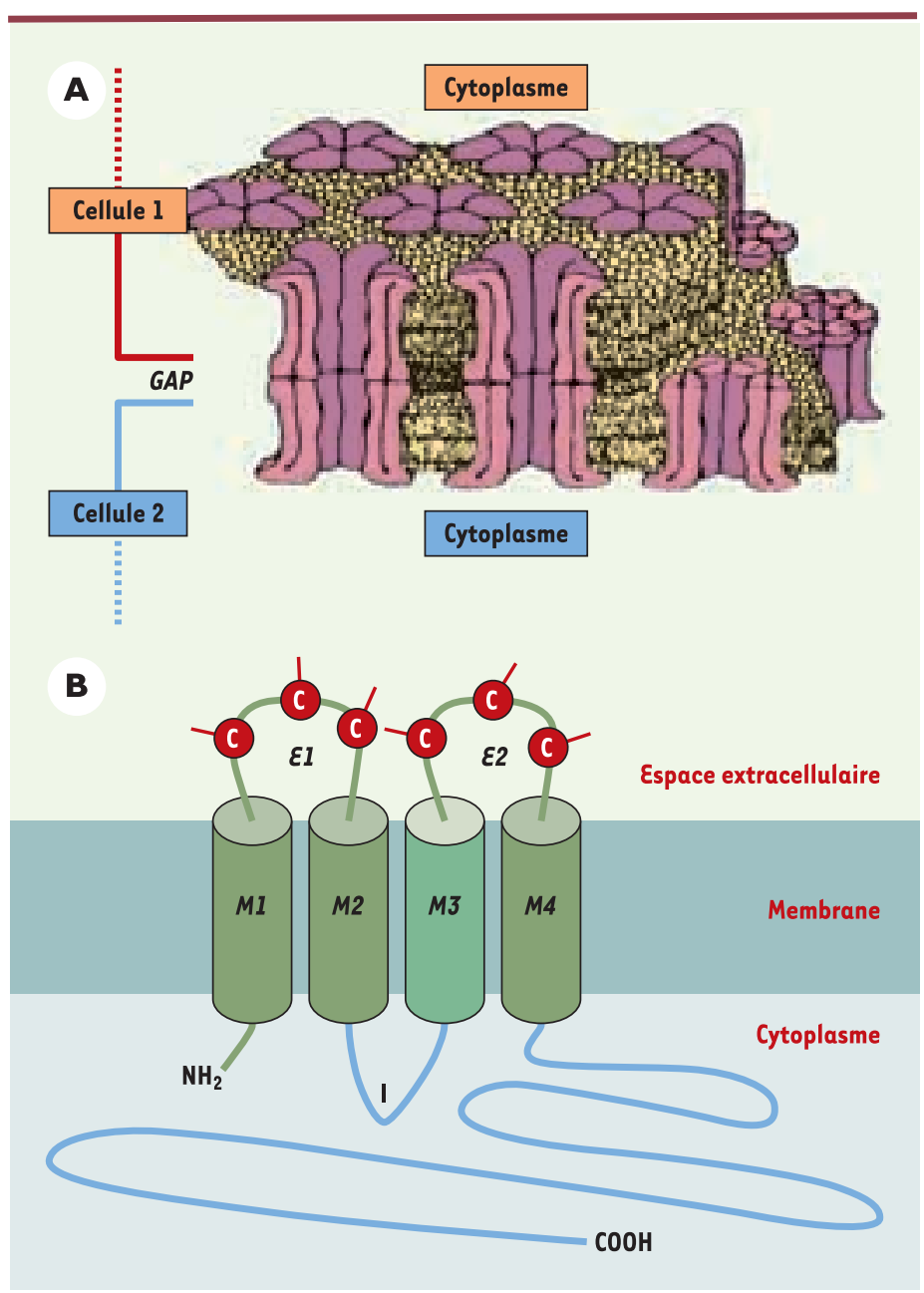
constituée par quatre domaines transmembranaires, Ml à M4. De par sa séquence, on admet que le domaine M3 (vert clair) est impliqué dans la formation du pore central par lequel transitent les molécules et les ions. Enfin, la partie extracellulaire comprend deux boucles ( $\varepsilon 1$ et $\varepsilon 2$ ) possédant chacune trois cystéines $(C)$ conservées chez tous les membres de la famille. Les trois cystéines participent à la formation de ponts disulfures intramoléculaires, qui confèrent aux connexines une conformation serrée et rigide leur permettant d'interagir étroitement avec les connexines des cellules adjacentes. Leur interaction étroite isole ainsi le canal intercellulaire du milieu extracellulaire. 
tétradécanoyl 12-13 phorbol acétate, extrait du croton, inhibait la communication jonctionnelle de cellules en culture $[7,8]$. Ces données, établissant un lien tangible entre les jonctions communicantes et la can- cérogenèse (Figure 3), furent confirmées sur d'autres modèles et permirent de considérer la plupart des promoteurs tumoraux comme étant des inhibiteurs de la communication jonctionnelle [10]. Il était tentant de

\begin{tabular}{|c|c|c|c|c|}
\hline $\begin{array}{l}\text { 'Type de } \\
\text { connexine }\end{array}$ & Viabilité & Expression tissulaire ${ }^{1}$ & $\begin{array}{l}\text { Phénotype des souris } \\
\text { homozygotes }^{-/-}\end{array}$ & $\begin{array}{l}\text { Rôle physiologique } \\
\text { proposé }\end{array}$ \\
\hline$C \times 26$ & - & $\begin{array}{l}\text { Glande mammaire, peau, } \\
\text { cochlée, foie, placenta }\end{array}$ & $\begin{array}{l}\text { Mort in utero (દ10 à દll) } \\
\text { Retard de croissance } \\
\text { de l'embryon }\end{array}$ & $\begin{array}{l}\text { Transfert du glucose entre } \\
\text { syncytiotrophoblastes I et II }\end{array}$ \\
\hline Cx32 & + & $\begin{array}{l}\text { Foie, cellules de Schwann, } \\
\text { oligodendrocytes, pancréas }\end{array}$ & $\begin{array}{l}\text { Augmentation de la sécrétion } \\
\text { d'amylase } \\
\text { Désorganisation des gaines } \\
\text { de myéline } \\
\text { Incidence accrue de tumeurs } \\
\text { hépatiques spontanées } \\
\text { ou induites chimiquement } \\
\text { Réduction de la mobilisation } \\
\text { orthosympathique du glucose } \\
\text { hépatique }\end{array}$ & $\begin{array}{l}\text { Régulation de la sécrétion } \\
\text { d'amylase } \\
\text { Homéostasie intracellulaire } \\
\text { des cellules de Schwann } \\
\text { Régulation de la différenciation } \\
\text { et de la croissance } \\
\text { des hépatocytes } \\
\text { Régulation de la diffusion } \\
\text { (entre autres) interhépatocytaire } \\
\text { d'inositol triphosphate }\end{array}$ \\
\hline$C \times 37$ & + & Endothélium, ovaire & $\begin{array}{l}\text { Absence d'ovulation } \\
\text { Développement anormal } \\
\text { du corps jaune }\end{array}$ & $\begin{array}{l}\text { Contrôle de la maturation } \\
\text { des follicules ovariens }\end{array}$ \\
\hline$C \times 40$ & + & Cœur, endothélium & $\begin{array}{l}\text { Diminution de la vitesse } \\
\text { de conduction atriale } \\
\text { et ventriculaire }\end{array}$ & $\begin{array}{l}\text { Régulation de la propagation } \\
\text { des potentiels d'action } \\
\text { dans les oreillettes et le tissu } \\
\text { conducteur du cœur } \\
\text { (faisceau de His, } \\
\text { réseau de Purkinje) }\end{array}$ \\
\hline Cx43 & - & $\begin{array}{l}\text { Cœur, nombreux autres } \\
\text { types cellulaires }\end{array}$ & $\begin{array}{l}\text { Mort périnatale } \\
\text { par malformation cardiaque } \\
\text { Retard d'ossification } \\
\text { et anomalies crâniofaciales } \\
\text { Diminution de la taille } \\
\text { des gonades } \\
\text { Arrêt de la folliculogenèse } \\
\text { ovarienne et de } \\
\text { la spermatogenèse }\end{array}$ & $\begin{array}{l}\text { Régulation de la migration } \\
\text { des cellules de la crête neurale } \\
\text { Régulation de la différenciation } \\
\text { osseuse } \\
\text { Organogenèse } \\
\text { de la reproduction } \\
\text { Contrôle de la maturation } \\
\text { des gamètes }\end{array}$ \\
\hline Cx45 & - & Cœur, muscle lisse & $\begin{array}{l}\text { Mort in utero ( } \varepsilon 10 \text { à } \varepsilon 11) \\
\text { Anomalies cardiaques, } \\
\text { formation altérée des vaisseaux } \\
\text { sanguins du placenta }\end{array}$ & $\begin{array}{l}\text { Morphogenèse cardiaque } \\
\text { et vasculaire }\end{array}$ \\
\hline Cx46 & + & Cristallin & Cataracte & $\begin{array}{l}\text { Homéostasie du cristallin } \\
\text { et intégrité des cristallines }\end{array}$ \\
\hline$C \times 50$ & + & Cristallin & $\begin{array}{l}\text { Cataracte } \\
\text { Microphtalmie }\end{array}$ & $\begin{array}{l}\text { Homéostasie du cristallin } \\
\text { Contrôle de la morphogenèse } \\
\text { du cristallin et de l'œil }\end{array}$ \\
\hline
\end{tabular}

Tableau I. Phénotypes observés chez des souris dont les gènes codant pour certains types de connexines ont été invalidés. ${ }^{1}$ Expression tissulaire chez les souris dont le gène n'a pas été invalidé (d'après [4]). 
voir l'inhibition de ce type de communication activement impliquée dans le processus de cancérogenèse, qui pourrait, lors d'une phase prétumorale, par des promoteurs tumoraux favoriser l'apparition du phénotype cancéreux en perturbant l'homéostasie tissulaire (Figure 3).

Par la suite, des anomalies de communication ont été observées dans de nombreuses lignées tumorigéniques. Selon les modèles, l'anomalie consistait en une perte de communication soit entre les cellules tumorigéniques (absence de communication homologue), soit avec les cellules normales uniquement (absence de communication hétérologue) (Figure 3) [9]. Le postulat de W.R. Loewenstein devenait un constat d'autant plus remarquable qu'il ne dépendait ni du type cellulaire étudié, ni de l'espèce animale considérée : constat dont l'uniformité n'était en réalité qu'apparente, les causes moléculaires affectant la fonction des connexines étant variées, directe (absence de transcription des gènes de connexines, de traduction de leur ARNm, d'un transport transcytoplasmique efficace de ces protéines ou de leur phosphorylation adéquate) ou indirectes (perte de la reconnaissance intercellulaire). La disparité des causes pouvait permettre d'attribuer à une altération de la communication jonctionnelle un rôle de dénominateur commun de la transformation tumorigénique (Figure 4). Si l'on a pu émettre l'hypothèse selon laquelle ce dénominateur commun n'était qu'un simple marqueur du phénotype tranformé, son implication allait en réalité s'avérer plus forte à la lumière des résultats obtenus à l'aide d'ADNc codant pour les connexines.

\section{L'implication «suppression tumorale»}

Les premiers $A D N c$ de connexine clonés furent ceux codant pour la Cx32 [11], la Cx43 [12] et la Cx26 [13]. La transfection de ces ADNc dans des cellules tumorales dépourvues de jonctions communicantes et l'induction de l'expression des connexines qui s'ensuit entraînent souvent une réduction du pouvoir tumorigénique ou du taux de réplication cellulaire [6]. Les connexines se comportent donc comme des régulateurs du phénotype, voire des suppresseurs de tumeur (Figure 4).

Toutefois, ce rôle suppresseur de tumeur est complexe, car la réexpression de la communication jonctionnelle n’entraîne pas systématiquement la perte du phénotype transformé. Par exemple, toutes les connexines testées confèrent aux cellules HeLa la capacité de communiquer, mais seule la $\mathrm{C} \times 26$, et parfois la $\mathrm{C} \times 43$, sont des suppresseurs de tumeur pour ces cellules [14]. L'équation «rétablissement de la communication jonctionnelle = suppression tumorale»n'est donc pas simple. D'autres paramètres entrent en jeu, puisque l'association va au-delà de la réinduction d'une communication et dépend du type de connexine réexprimé. La littérature scientifique à ce sujet semble aller dans

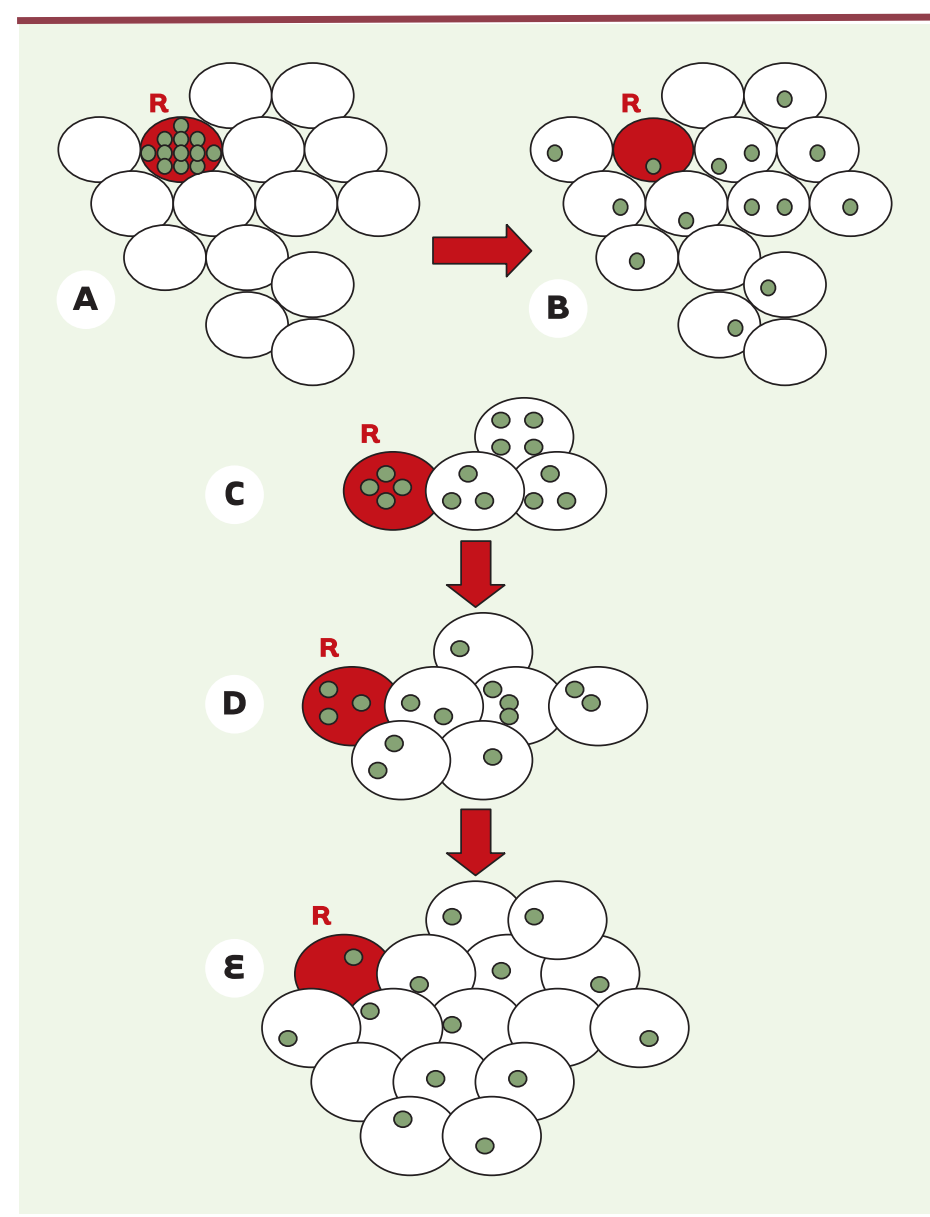

Figure 2. Hypothèse de l'implication des jonctions communicantes dans le contrôle de la réplication cellulaire. L'hypothèse émise par W.R. Loewenstein [6] suggère que la multiplication cellulaire serait contrôlée par la concentration intracellulaire d'un facteur de croissance cytoplasmique (cercle vert). $\boldsymbol{A}$, B. La diffusion, par l'intermédiaire des jonctions communicantes, de ce facteur depuis la cellule émettrice rouge «R» vers les cellules voisines l'empêcherait d'atteindre, dans chacune des cellules, la concentration seuil nécessaire à l'induction de leur réplication : le nombre de cellules est ainsi identique en $\boldsymbol{A}$ et en $B$. De même, une cellule «R» (cellule rouge) serait capable de proliférer tant que le facteur de croissance qu'elle produit atteint une concentration seuil donnée dans le cytoplasme. Malgré la diffusion du facteur depuis la cellule mère $\ll R »$ vers les cellules filles (cellules blanches), ces cellules seraient capables de proliférer tant que cette concentration demeure élevée. Le nombre de cellules passerait ainsi d'un stade $C$ à un stade $D$ pour atteindre un stade stationnaire $\varepsilon$ pour lequel la concentration intracellulaire du facteur de croissance est insuffisante, par effet de dilution, pour stimuler la prolifération de ce groupe de cellules. 
ce sens, la suppression tumorale est surtout obtenue à la suite de la réinduction de l'expression de la connexine d'origine (celle normalement présente dans le tissu avant cancérisation, ou dans la lignée cellulaire avant transformation (Tableau II).

\section{Quelle(s) fonction(s) pour les connexines?}

L'élucidation de l'effet suppresseur de tumeur par les connexines dépend d'une meilleure connaissance de leur biologie; or il faut admettre que nous ne savons

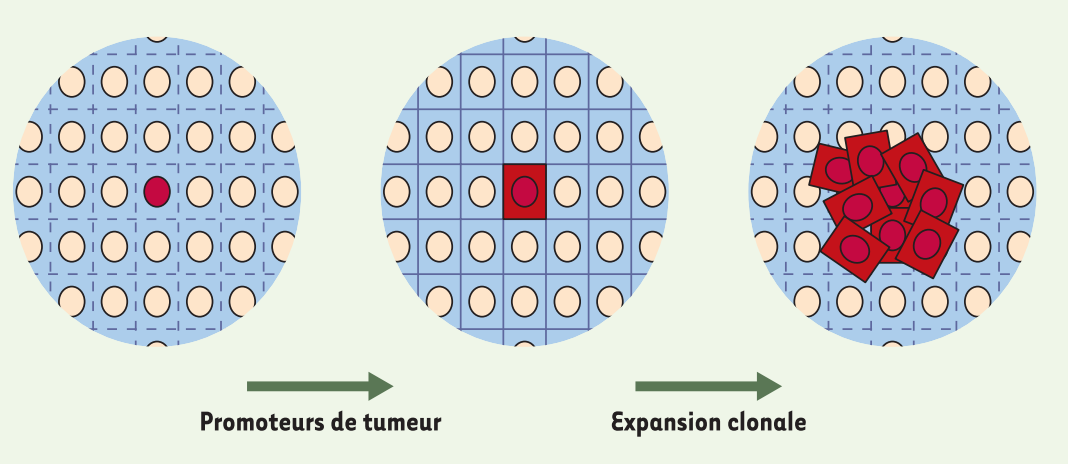

Figure 3. Implication des jonctions communicantes au cours de la cancérogenèse. Au sein d'une population cellulaire, une cellule génotypiquement transformée (noyau rouge) n'exprimerait pas son phénotype si toutes les cellules communiquent entre elles (traits pointillés). Les promoteurs de tumeur favoriseraient l'expression du phénotype transformé (cytoplasme rouge) en inhibant le réseau de communication jonctionnelle intercellulaire. L'expression de son phénotype transformé et le taux accru de réplication cellulaire qui s'ensuivrait conduiraient alors à la prolifération clonale de la cellule transformée [9]. Noter l'absence de communication (traits pleins) entre les cellules exprimant le phénotype aberrant et les cellules normales (bleues) environnantes qui empêcherait l'arrêt de la prolifération. Cette absence de communication pourrait être sélective (uniquement entre les cellules transformées et les cellules normales) ou affecter chacune des cellules transformées (comme présenté sur la figure).

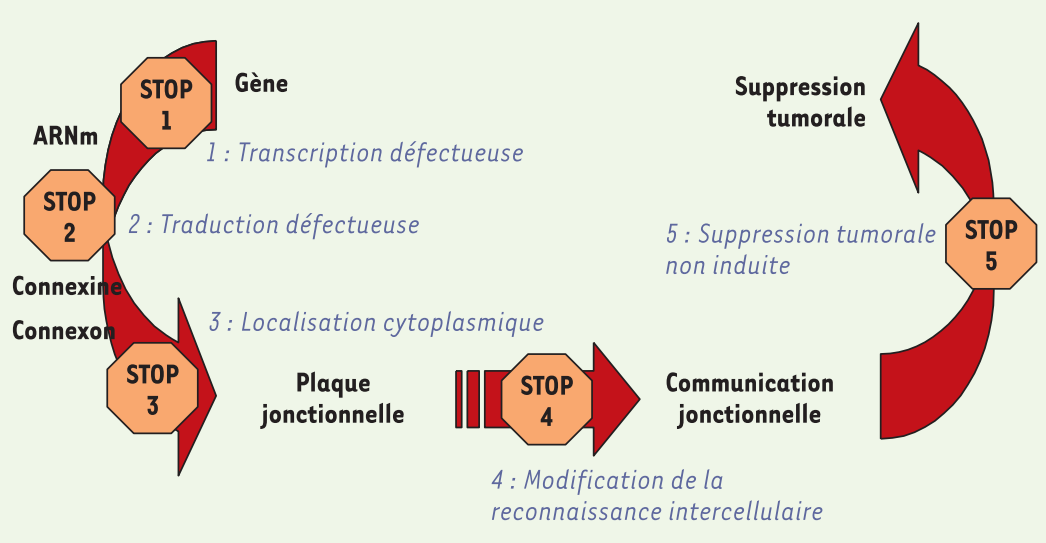

Figure 4. Communication jonctionnelle intercellulaire et suppression tumorale. Les données moléculaires obtenues dans différentes lignées de cellules tumorales ou, in vivo, dans des modèles animaux, ont montré que chacune des étapes conduisant à l'établissement de la communication jonctionnelle peut être perturbée (en bleu sur le schéma) et provoquer la perte de la communication jonctionnelle. Des données plus récentes ont montré que le rétablissement de la communication jonctionnelle par transfection d'ADNc de connexine dans des lignées tumorales non communicantes n'induisait pas automatiquement un effet suppresseur de tumeur (étape 5) : l'induction de cet effet peut en effet dépendre du type de connexine dont l'expression a été réinduite. presque rien de la nature

de la communication qu'elles modulent ni comment elles participent au processus transformant. Peu de molécules transférées par l'intermédiaire des jonctions communicantes ont été identifiées. Parmi les quelques molécules transmissibles identifiées, certaines, comme l'adénosine diphosphate impliquée dans des voies métaboliques fondamentales, ne permettent pas de supposer un rôle spécifique des connexines dans la cancérogenèse [18]. Certes, la perméabilité à des seconds messagers appuie l'idée d'un rôle des connexines dans le maintien de l'homéostasie tissulaire, mais sans plus [19]. La microinjection de sondes fluorescentes a seulement montré que les connexines sont douées de perméabilités différentes [20], rendues plus subtiles par leur capacité à former des connexons hétéromériques ${ }^{1}$ [21].

Si la réinduction de la communication jonctionnelle n'aboutit pas toujours à une normalisation du phénotype, nous avons également observé que des mutations réduisant la capacité fonctionnelle de la Cx26 n'augmentent pas le pouvoir tumorigénique des cellules HeLa [22]. Ces résultats suggèrent un cumul de fonctions pour les connexines: celle de modulateurs de la communication intercytoplasmique et celle de régulateurs de la prolifération cellulaire. Cette hypothèse est corroborée par une localisation des connexines parfois incompatible avec un rôle de médiation de la communication jonctionnelle : une étude rapporte, par exemple, une localisation nucléaire - et non pas membranaire - de la Cx43 dans des cellules transformées par des oncogènes [23]; de même, la suppression tumorale induite par la $\mathrm{C} \times 43$ dans des cellules de glioblastomes n'est

${ }^{1}$ Connexons hétéromériques: constitués de connexines de type différent. 
pas associée à un gain de communication jonctionnelle, mais plutôt à la localisation cytoplasmique de la protéine [24]. Bien qu'éparses, ces données suggèrent que les connexines possèderaient d'autres fonctions que celle de simple médiateur de la communication jonctionnelle (Tableau III). De plus, les interactions directes qui ont été récemment observées entre la $\mathrm{C} \times 43$ et d'autres protéines (submembranaires ou cytoplasmiques) font paraître les connexines non plus seulement comme de simples éléments constitutifs des jonctions communicantes [25], mais aussi comme des éléments pouvant être impliqués dans différentes voies de signalisations.

\section{Questions en suspens}

Malgré ces considérations, l'hypothèse de W.R. Loewenstein demeure valable. Cependant, il reste à définir le rôle exact des connexines dans le processus cancéreux, et un certain nombre de questions demeurent donc sans réponse. Le rôle des connexines est-il lié au seul maintien de la communication jonctionnelle? Si oui, quels sont les facteurs régulateurs de la croissance dont elles modulent le transfert ? À quel(s) stade(s) du processus cancéreux interviennentelles? Y sont-elles impliquées pour la régulation d'autres processus comme l'apoptose [26]? Ont-elles un autre rôle que de moduler la communication intercellulaire? Existe-t-il des interactions entre elles et d'autres protéines qui contrôleraient le cycle cellulaire? Les études dont a fait l'objet la $\mathrm{C} \times 43$ portent les questions à un niveau plus moléculaire: l'expression de la Cx43 réduit celle de kinases liées au cycle celIulaire [27]; pourquoi cette connexine est-elle nucléaire lorsque certains oncogènes sont activés? Sa localisation cytoplasmique est-elle corrélée à la perte de tumorigénicité des cellules de gliomes? Les interactions entre la Cx43 et d'autres protéines participent-elles au contrôle de la multiplication cellulaire?

${ }^{1}$ Données non publiées.
Résoudre ces interrogations est d'importance, en particulier dans l'hypothèse d'une utilisation thérapeutique des connexines. En effet, la communication jonctionnelle permet d'obtenir l'effet de proximité (bystander effect) observé dans certaines thérapies expérimentales employant des gènes suicides tels que le gène HSV-tk (herpes simplex virus-thymidine kinase) [28] (voir Encadré). Si elles étaient rendues plus efficaces chez l'homme, ces stratégies thérapeutiques présenteraient l'avantage

\begin{tabular}{|c|c|c|c|}
\hline \multicolumn{4}{|c|}{ Connexine } \\
\hline Type cellulaire & Endogène & Transfectée & Tumorigénicité \\
\hline Hépatome (foie) ${ }^{1}$ & Cx32 & Cx32* & $\searrow$ \\
\hline Fibroblastes $^{2}$ & Cx43 & Cx43 & $\searrow$ \\
\hline \multirow[t]{4}{*}{ Cellule HeLa (col de l'utérus) ${ }^{1}$} & $C \times 26$ & $C \times 26$ & $\searrow$ \\
\hline & $\mathrm{Cx} 43$ & $\mathrm{C} \times 43 \star \star$ & $\searrow$ \\
\hline & & Cx32 & $\rightarrow$ \\
\hline & & Cx40 & $\rightarrow$ \\
\hline \multirow[t]{3}{*}{ Gliome $^{3}$} & Cx43 & Cx43 & $\searrow$ \\
\hline & $\mathrm{Cx} 30$ & Cx30*** & $\searrow$ \\
\hline & & Cx32* & $\searrow$ \\
\hline \multirow[t]{2}{*}{ Carcinome $(\operatorname{sein})^{1}$} & $C \times 26$ & $C \times 26$ & $\searrow$ \\
\hline & Cx43 & Cx43 & $\searrow$ \\
\hline
\end{tabular}

Tableau II. Suppression tumorale et expression spécifique de connexines. L'induction de la synthèse de connexines par transfection d'ADNc n'inhibe pas systématiquement la tumorigénicité de cellules d'origine tumorale. Cette inhibition coïnciderait avec la réexpression de la connexine originellement exprimée dans le tissu normal (en rouge), contrairement aux autres connexines

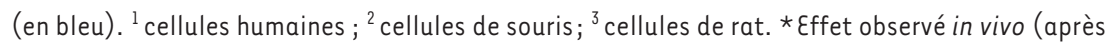
injection sous-cutanée administrée à des souris immunodéprimées), mais pas in vitro; ** effet non observé pour tous les clones; ${ }^{\star \star \star}$ seule la prolifération in vitro a été testée [14-17].

\begin{tabular}{llll}
\hline Connexines & Effets/localisation & Cellules/tissus & Références \\
Cx26 & Induction de l'adhérence cellulaire & HeLa ${ }^{1}$ & \\
Cx43 & Inhibition de la tumorigénicité & \\
& par localisation cytoplasmique & Gliome & Cellules épithéliales de foie \\
& Localisation nucléaire & transformées par src ou neu & [23] \\
& Interaction avec Z0-1, src & & [25] \\
& (non exhaustif) & & Ind] \\
& Induction de la capacité d'invasion & HeLa &
\end{tabular}

Tableau III. Localisation et effets de connexines (Cx26 et Cx43) apparemment non liés à leur fonction de modulateur de la communication jonctionnelle intercellulaire. 
d'administrer, dans le cas de tumeurs à faible pouvoir métastatique, des chimiothérapies localisées moins lourdes à supporter pour le patient [17]. Jusqu'à présent, ces thérapies géniques n'ont pas eu le succès escompté [29] : hormis le fait que les phases d'essai ont souvent concerné des patients au stade ultime de la maladie, il semble que les échecs obtenus proviennent aussi d'un mauvais transfert intratumoral des gènes suicides [30]. D'après nos résultats, l'effet de proximité dû à la communication jonctionnelle pourrait suppléer à ce défaut [31]; mais la fonction aberrante des connexines dans les cellules cancéreuses risque de s'opposer à la mise en place d'un effet de proximité conséquent. Si plus de données concernant les connexines dans les tumeurs humaines étaient disponibles, nous pourrions identifier les types de tumeurs qui, pourvues en connexines, seraient susceptibles d'être ciblées par la thérapie génique suicide du gène HSV-tk. L'application thérapeutique des connexines pourrait en outre favoriser l'action de substances chimiothérapeutiques classiques [32].

\section{Conclusions et perspectives}

Si de nombreux travaux vont dans le sens d'une implication des connexines dans la cancérogenèse, les données sont cependant encore plus de l'ordre de la suggestion, et n'élucident pas le rôle véritable des connexines dans ce processus. Jusqu'à présent, les travaux sur les connexines se sont peu focalisés sur l'étude de cancers sporadiques chez l'homme: il a bien été montré que la régulation moléculaire de la communication jonctionnelle est corrélée à diverses étapes de transformation in vitro ou de progression tumorale chez l'animal, mais les données chez l'homme (Tableau IV) sont encore trop éparses ou contradictoires pour pouvoir en tirer une conclusion générale quant à une implication certaine des connexines dans la cancérogenèse humaine. Une analyse de l'expression et de la localisation des connexines tenant compte de l'état de progression des tumeurs serait profitable pour cerner davantage le rôle tenu par les jonctions communicantes dans la cancérogenèse.

L'analyse moléculaire précise des connexines in situ pourrait également avoir des répercussions dans le domaine de la thérapie antitumorale, puisque les jonctions communicantes seraient capables d'améliorer l'efficacité de traitements chimiothérapeutiques en permettant probablement la diffusion de signaux apoptotiques $[32,41]$. De plus, des données suggèrent que l'effet de proximité peut être amplifié par des traitements capables d'augmenter la communication jonctionnelle $[42,43]$. Chez l'animal, de tels résultats ont été obtenus après injection intrapéritonéale d'acide rétinoïque, d'apigénine ou de lovastatine. Cela pourrait être les prémices d'une «manipulation pharmacologique», à but thérapeutique, des jonctions communicantes [43]. 
L'autre aspect des connexines méritant d'être étudié est celui de la spécificité de leur action de suppresseur de tumeur (Tableau II). Autrement dit, l'effet suppresseur de tumeur observé après transfection de gènes de connexines dans des cellules tumorales ne se rencontrerait que pour les connexines normalement présentes dans le tissu avant cancérisation. Pour vérifier cette hypothèse, il faudrait réexprimer des connexines spécifiques dans des lignées tumorales de différentes origines, en prenant garde à ne pas sélectionner les clones à forte croissance, susceptibles d'être moins pourvus en connexines si celles-ci sont effectivement impliquées dans la régulation négative de la croissance cellulaire. Une fois ces clones obtenus, l'étude des mécanismes moléculaires de la suppression tumorale induite par l'expression spécifique de connexines serait envisageable.

On le voit, les sujets d'étude ne manquent pas et débordent le seul cadre des jonctions communicantes. Les données accumulées suggèrent que ces jonctions sont une pièce d'un puzzle, ou plutôt qu'elles font partie d'un réseau de régulation du phénotype cellulaire. Elles seraient à un carrefour physiologique isolé artificiellement des autres systèmes fondamentaux de la cellule du fait de notre propension à compartimenter les phénomènes. La compréhension de l'action des connexines dans la cellule dépendra de l'intégration d'une étude moléculaire spécifique dans une vision élargie des phénomènes impliqués. II convient de ne pas perdre la vision de la forêt par l'étude approfondie de l'écorce d'un seul arbre. En d'autres termes, il ne faudrait pas que nous soyons comme Sganarelle affirmant: «Nous autres grands médecins, nous connaissons d'abord les choses $^{1}$.»

\begin{tabular}{|c|c|c|}
\hline $\begin{array}{l}\text { Type de tumeur } \\
\text { Tissu }\end{array}$ & Connexines étudiées & Références \\
\hline $\begin{array}{l}\text { Dysplasie } \\
\text { Col de l'utérus }\end{array}$ & Expression réduite de $\mathrm{Cx} 43$ & [33] \\
\hline $\begin{array}{l}\text { Adénocarcinomes } \\
\text { Côlon }\end{array}$ & $\begin{array}{l}\text { Mutations de l'extrémité carboxyle cytoplasmique de Cx43 } \\
\text { et expression du mutant dans les zones invasives }\end{array}$ & [34] \\
\hline $\begin{array}{l}\text { Carcinomes } \\
\text { Estomac }\end{array}$ & Perte du couplage ionique (pas d'études moléculaires) & Cité dans $[6,17]$ \\
\hline $\begin{array}{l}\text { Hépatocarcinomes } \\
\text { Foie }\end{array}$ & $\begin{array}{l}\text { Diminution du couplage } \\
\text { Localisation cytoplasmique de } C \times 32 \\
\text { Expression anormale et cytoplasmique de } C \times 43\end{array}$ & {$[35]$} \\
\hline $\begin{array}{l}\text { Carcinomes } \\
\text { Larynx }\end{array}$ & $\begin{array}{l}\text { Détection hétérogène des } \mathrm{C} \times 26, \mathrm{C} \times 30, \mathrm{C} \times 43 \text { (alternance de zones } \\
\text { d'expression et de zones sans expression) }\end{array}$ & {$[36]$} \\
\hline $\begin{array}{l}\text { Tissu normal } \\
\text { Prostate }\end{array}$ & $\begin{array}{l}\text { Expression de } \mathrm{C} \times 32 \text { (seule détectable) } \\
\text { Expression de } \mathrm{C} 32 \text { et de } \mathrm{Cx} 43 \text { (dans respectivement } 65 \% \text { et } 87 \% \\
\text { des échantillons) }\end{array}$ & $\begin{array}{l}{[37]} \\
{[38]}\end{array}$ \\
\hline $\begin{array}{l}\text { Tumeurs } \\
\text { Prostate }\end{array}$ & $\begin{array}{l}\text { Coexpression membranaire (tumeurs différenciées) ou cytoplasmique } \\
\text { (tumeurs indifférenciées) de Cx32 et de Cx43 } \\
\text { Perte d'expression de } C \times 32 \text { seule, de } C \times 43 \text { seule, des deux Cx } \\
\text { (dans respectivement } 38 \%, 65 \%, 28 \% \text { des échantillons) }\end{array}$ & [37] \\
\hline $\begin{array}{l}\text { Carcinomes } \\
\text { Sein }\end{array}$ & Perte d'expression de $\mathrm{C} \times 43$ & [39] \\
\hline $\begin{array}{l}\text { Carcinomes } \\
\text { Tête et cou }\end{array}$ & Diminution d'expression de $C \times 31.1$ & {$[40]$} \\
\hline
\end{tabular}

Tableau IV. Expression et caractéristiques des connexines révélées par des études in situ dans des tumeurs humaines. 


\section{SUMMARY}

Gap junctions and cancer: implications and perspectives

Gap junctions are made of intercellular channels which permit the diffusion from cytoplasm to cytoplasm of small hydrophilic molecules (<1 $200 \mathrm{Da})$ such as ions, sugars, aminoacids, nucleotides, second messengers (calcium, inositol triphosphate, etc.). Since their discovery in the early sixties, several groups have described the loss of their function in cancer cells. The accumulation of such data led to the hypothesis that gap junctions are involved in the carcinogenesis process. This assumption has been confirmed by data establishing that gap junctional intercellular communication is inhibited by most of the tumor promoters and that the restoration of such a communication, by transfection of cDNAs encoding gap junction proteins (connexins), inhibits the aberrant growth rates of tumorigenic cells. Despite these important informations, several fundamental questions remain still open. First, we do not know how gap junctions mediate such a tumor suppressor effect and whether it may depend either on the cell type or on the connexin type. Moreover, most of the data concerning a possible involvement of gap junctions in carcinogenesis have been obtained from in vitro and animal models. The very few results which have been currently collected from human tumors are not sufficient to have a clear idea concerning the real involvement of gap junctions in sporadic human cancers. These points as well as other unresolved questions about the role of gap junctional intercellular communication in carcinogenesis are mentionned. To bring some answers, some prospects are proposed with the objective to use gap junctions for increasing the effect of anticancer therapies. $\diamond$

\section{RéFÉRENCES}

1. Abarcrombie M, Heaysman EM. Social behavior of cells in monolayering of fibroblasts. Exp Cell Res $1954 ; 6: 293$.

2. Makowski L, Caspar DL, Phillips WC, Goodenough DA. Gap junction structures. II. Analysis of the $\mathrm{X}$-ray diffraction data. J Cell Biol 1977 ; $74: 629-45$.
6. Loewenstein WR. Junctiona intercellular communication and the control of growth. Biochim $560: 1-65$. Biophys Acta 1979 ;
7. Murray AW, Fitzgerald DJ. Tumor promoters inhibit metabolic cooperation in cocultures of epidermal and $3 T 3$ cells. Biochem Biophys Res Comm 1979; 91: 395-401.

8. Yotti LP, Chang CC, Trosko JE. Elimination of metabolic cooperation in Chinese hamster cells by a tumor promoter. Science 1979 ; 206 : 1089-91.

9. Yamasaki H, Naus CCG. Role of connexin genes in growth control. Carcinogenesis 1996 ; $17: 1199-213$.

10. Budunova IV, Williams GM. Cell culture assays for chemicals with tumorpromoting or tumorinhibiting activity based on the modulation of intercellular communication. Cell Biol Toxicol 1994 ; 10: 71-116.

11. Paul DL. Molecular cloning of cDNA for rat liver gap junction protein. J Cell Biol 1986; 103: 123-34.

12. Beyer $\varepsilon C$, Paul DL, Goodenough DA. Connexin 43 : a protein from rat heart homologous to a gap junction protein from liver. J Cell Biol 1987 ; 105: 2621-9.

13. Zhang JT, Nicholson BJ. Sequence and tissue distribution of a second protein of hepatic gap junctions, Cx26, as deduced from its cDNA. J Cell Biol 1989 ; 109: 3391-401.

14. Mesnil M, Krutovskikh V, Piccoli C, et al. Negative growth control of HeLa cells by connexin genes : connexin species specificity. Cancer Res 1995 ; 55 : 629-39.

15. Bond SL, Bechberger JF, Khoo NK, Naus CCG. Transfection of C6 glioma cells with connexin 32 : the effects of expression of a nonendogenous gap junction protein. Cell Growth Differ 1994 ; $5: 179-86$.
16. Princen F, Robe P, Gros D, et al. Rat gap junction connexin-30 inhibits proliferation of glioma cell lines. Carcinogenesis 2001; $22: 507-13$.

17. Mesnil M, Yamasaki H. Bystander effect in herpes simplex virus-thymidine kinase/ganciclovir cancer gene therapy: role of gapjunctional intercellular communication. Cancer Res $2000 ; 60$ : 3989-99.

18. Goldberg GS, Lampe PD, Sheedy D, Stewart CC, Nicholson BJ, Naus CCG. Direct isolation and analysis of endogenous transjunctional ADP from Cx43-transfected C6 glioma cells. Exp Cell Res 1998 ; $239: 82-92$.

19. Saez JC, Connor JA, Spray DC, Bennett MVL. Hepatocyte gap junctions are permeable to the second messenger, inositol 1, 4, 5-triphosphate, and to calcium ions. Proc Natl Acad Sci USA 1989 ; $86: 2708-12$

20. Elfgang $C$, Eckert R, Lichtenberg-Fraté $\mathrm{H}$, et al. Specific permeability and selective formation of gap junction channels in connexin-transfected HeLa cells. J Cell Biol 1995 ; 129 : 805-17.

21. Kumar NM, Gilula NB. The gap junction communication channel. Cell $1996 ; 84$ : 381-8.

22. Duflot-Dancer A, Mesnil M, Yamasaki H. Dominantnegative abrogation of connexin-mediated cell growth control by mutant connexin genes. Oncogene 1997 ; 15 : 2151-8.

23. De Feijter AW, Matesic DF, Ruch RJ, Guan X, Chang CC, Trosko JE. Localization and function of the connexin 43 gap-junction protein in normal and various oncogene-expressing rat liver epithelial cells. Mol Carcinogen 1996 ; 16 : 203-12. 
24. Huang RP, Fan $Y$, Hossain MZ, Peng A, Zeng ZL, Boynton AL. Reversion of the neoplastic phenotype of human glioblastoma cells by connexin 43 (Cx43). Cancer Res 1998 ; 58 : 5089-96.

25. Hervé JC, Duthé F, Plaisance I, Bahbouhi B, Sarrouilhe D. The partner proteins of connexins on the track. Recent Res Dev Physiol 2003; 1: 103-17.

26. Trosko JE, Goodman JI. Intercellular communication may facilitate apoptosis: implications for tumor promotion. Mol Carcinogen $1994 ; 11: 8-12$.

27. Chen SC, Pelletier DB, Ao P, Boynton AL. Connexin 43 reverses the phenotype of transformed cells and alters their expression of cyclin/cyclin-dependent kinases. Cell Growth Differ $1995 ; 6: 681-90$.

28. Mesnil M, Piccoli C, Tiraby G, Willecke K, Yamasaki H. Bystander killing of cancer cells by herpes simplex virus thymidine kinase gene is mediated by connexins. Proc Natl Acad Sci USA 1996; 93 : 1831-5.

29. Klatzmann D, Valery CA, Bensimon $G$, et al. A phase I/II study of herpes simplex virus typel thymidine kinase «suicide» gene therapy for recurrent glioblastoma. Study group on gene therapy for glioblastoma. Hum Gene Ther $1998 ; 9: 2595-604$.

30. Ram Z, Culver KW, Oshiro $\varepsilon M$, et al. Therapy of malignant brain tumors by intratumoral implantation of retroviral vectorproducing cells. Nat Med 1997 ; 3 : 1354-61.
31. Mesnil M, Yamasaki H. La possibilité d'une thérapie génique antitumorale amplifiée par l'induction de la communication jonctionnelle intercellulaire. Med Sci (Paris) 1996 ; 12 : 1435-8.

32. Huang RP, Hossain MZ, Huang R, Gano J, Fan Y, Boynton AL. Connexin 43 (cx43) enhances chemotherapy-induced apoptosis in human glioblastoma cells. Int J Cancer 2001 ; 92 : 130-8.

33. King TJ, Fukushima LH, Hieber AD, Shimabukuro KA, Sakr WA, Bertram JS. Reduced levels of connexin 43 in cervical dysplasia: Inducible expression in a cervical carcinoma cell line decreases neoplastic potential with implications for tumor progression. Carcinogenesis 2000 ; 21 : 1097-109.

34. Dubina MV, latckii NA, Popov DE, Vasiliev SV, Krutovskikh VA. Connexin 43, but not connexin 32 , is mutated at advanced stages of human sporadic colon cancer. Oncogene 2002 ; 21 : 4992-6.

35. Krutovskikh V, Mazzoleni G, Mironov N, et al. Altered homologous and heterologous gapjunctional intercellular communication in primary human liver tumors associated with aberrant protein localization but not gene mutation of connexin 32. Int J Cancer 1994 ; $56: 87-94$.
36. Schneider B, Teschner M, Sudermann T, Pikula B, Lautermann J. Expression of gap junction proteins (connexin 26, 30, 32, 43) in normal mucosa, hyperkeratosis and carcinoma of the human larynx. J Otorhinolaryngol Relat Spec 2002 ; 64 : 324-9.

37. Mehta PP, Perez-Stable C, Nadji M, Mian M, Asotra K, Roos BA. Suppression of human prostate cancer cell growth by forced expression of connexin genes. Dev Genet 1999 ; 24: 91-110.

38. Habermann H, Ray V, Habermann W, Prins GS. Alterations in gap junction protein expression in human benign prostatic hyperplasia and prostate cancer. J Urol 2002 ; 167 : 655-60.

39. Laird DW, Fistouris $P$, Batist $G$, et al. Deficiency of connexin 43 gap junctions is an independent marker for breast tumors. Cancer Res 1999; 59: 4104-10.

40. Al Moustafa AE, AlaouiJamali MA, Batist G, et al. Identification of genes associated with head and neck carcinogenesis by cDNA microarray comparison between matched primary normal epithelial and squamous carcinoma cells. Oncogene 2002 ; 21 : 2634-40.
41. Krutovskikh VA, Piccoli C, Yamasaki H. Gap junction intercellular communication propagates cell death in cancerous cells. Oncogene $2002 ; 27$ : 1989-99.

42. Park Jy, Elshami AA, Amin K, Rizk N, Kaiser LR, Albelda SM. Retinoids augment the bystander effect in vitro and in vivo in herpes simplex virus thymidine kinase/ganciclovirmediated gene therapy. Gene Ther 1997 ; 4 : 909-17.

43. Touraine RL, Ishii-Morita $H$, Ramsey WJ, Blaese RM. The bystander effect in the HSVtk/ganciclovir system and its relationship to gap junctional communication. Gene Ther 1998 ; 5: 1705-11. 